\title{
Lesão variante de Maisonneuve com luxação tibiofibular proximal*
}

\section{Maisonneuve Variant Lesion with Proximal Tibiofibular Dislocation}

\author{
Jonatas Brito Alencar Neto ${ }^{10}$ Maria Luzete Costa Cavalcante ${ }^{2}$ Luiz Holanda Pinto Neto ${ }^{1}$
} Igor Freitas de Lucena ${ }^{1}$ Renackson Jordelino Garrido ${ }^{2}$ Pedro Henrique Messias da Rocha ${ }^{2}$

${ }^{1}$ Departamento de Ortopedia e Traumatologia, Instituto Doutor José Frota, Fortaleza, CE, Brasil

2 Departamento de Ortopedia, Universidade Federal do Ceará, Hospital Walter Cantídio, Fortaleza, CE, Brasil

Address for correspondence Jonatas Brito Alencar Neto, Rua Joaquim Nabuco, 1850, Aldeota, Fortaleza, Ceará, CEP: 60125-120, Brasil (e-mail: jonatasbrito19@hotmail.com).

Rev Bras Ortop 2019;54:339-342.

\section{Resumo \\ Palavras-chave \\ - traumatismos do tornozelo \\ - luxações articulares \\ - procedimentos cirúrgicos operatórios}

\section{Abstract \\ Keywords \\ - ankle injuries \\ - joint dislocations \\ - surgical procedures, operative}

A lesão de Maisonneuve é uma entidade rara, correspondendo a 7\% das fraturas de tornozelo. Uma de suas variantes inclui a lesão da sindesmose tibiofibular distal com luxação tibiofibular proximal, tornando-a uma injúria ainda mais incomum. O presente artigo apresenta o caso de um paciente de 34 anos admitido na emergência de traumato-ortopedia de hospital terciário com dor e edema em membro inferior esquerdo, após trauma esportivo. A radiografia do joelho evidenciou luxação anterolateral da articulação tibiofibular proximal com subluxação lateral do tornozelo, sem sinais de fratura. Após avaliação tomográfica do membro, confirmou-se o diagnóstico de uma lesão variante de Maisonneuve com luxação tibiofibular proximal. Os autores descrevem o caso, abordando aspectos clínicos, radiográficos e cirúrgicos desta variante.

Maisonneuve injury is a rare entity accounting for $7 \%$ of all ankle fractures. One of its variants includes a proximal tibiofibular dislocation, which is an even more unusual injury. The present article reports a case of a 34-year-old male patient admitted to the emergency room with edema in the lower left limb after a sports trauma. An X-ray of the knee revealed anterolateral subluxation of the proximal tibiofibular joint, with no signs of fracture. After the evaluation of the member, the diagnosis of a lesion variant of Maisonneuve with proximal tibiofibular dislocation was confirmed. The authors describe this case, addressing clinical, radiological and surgical aspects of this variant.

Trabalho feito na Instituição Doutor José Frota, Fortaleza, Ceará, Brasil.

(D)Jonatas Brito Alencar Neto's ORCID is https://orcid.org/0000-00033318-5067.

received January 4, 2018 accepted July 2, 2018
DOI https://doi.org/ 10.1055/s-0039-1692625. ISSN 0102-3616.
Copyright $\odot 2019$ by Sociedade Brasileira License terms de Ortopedia e Traumatologia. Published by Thieme Revnter Publicações Ltda, Rio de Janeiro, Brazil 


\section{Introdução}

As lesões ligamentares do tornozelo ligadas ao esporte são frequentemente diagnosticadas nas emergências traumatológicas, principalmente na faixa etária dos 15 aos 35 anos. $^{1}$ As fraturas são mais prevalentes nos traumas desta região, como fraturas tanto do maléolo medial quanto do lateral. ${ }^{2}$ Desta forma, acaba sendo incomum a disjunção simultânea da articulação tibiofibular proximal e da sindesmose distal sem qualquer tipo de fratura, seja da tíbia ou da fíbula. ${ }^{2}$

A lesão de Maisonneuve, primeiramente descrita em 1840 pelo cirurgião Jules Germain François Maisonneuve, corresponde a $7 \%$ de todas as fraturas do tornozelo. ${ }^{3} 0$ mecanismo de trauma envolve uma rotação externa associada a pronação do tornozelo, o que leva a lesão do ligamento tibiofibular distal, do complexo sindesmótico e fratura do terço proximal da fíbula. Uma das variantes desta lesão corresponde a uma luxação concomitante na articulação tibiofibular proximal e da sindesmose distal do tornozelo na ausência de fratura do terço proximal da fíbula. ${ }^{3}$

Apenas quatro casos desta lesão rara foram relatados até este ano. ${ }^{4}$ O objetivo do presente artigo é apresentar um caso original de um paciente com uma lesão variante de Maisonneuve. Neste caso, uma lesão da sindesmose tibiofibular e luxação tibiofibular proximal.

\section{Relato de Caso}

Um paciente de 34 anos foi admitido na emergência após trauma no membro inferior esquerdo durante uma partida de futebol. Apresentava-se com dor intensa no tornozelo e joelho esquerdos após o trauma, com incapacidade de deambular. 0 exame clínico revelou edema na topografia do maléolo lateral esquerdo e na face lateral do joelho esquerdo. Sem alterações neurológicas ou vasculares.

Foram feitas radiografias que revelaram subluxação lateral do tornozelo esquerdo e aumento do espaço claro medial, associados à luxação anterolateral da articulação tibiofibular proximal ( - Fig. 1), sem evidências de fratura. Procedeu-se com estudo tomográfico da articulação do joelho, confirmando uma variante da lesão de Maisonneuve com luxação anterolateral da articulação tibiofibular proximal (- Fig. 2).

O paciente do estudo foi submetido a redução anatômica cirúrgica sob raquianestesia. Utilizou-se o acesso lateral curvilíneo ao joelho iniciando no côndilo lateral do fêmur em direção à borda anterior da porção proximal da fíbula. $O$ nervo fibular comum foi identificado e afastado delicadamente. A articulação tibiofibular foi identificada e o hematoma da lesão foi drenado, realizando-se a redução e fixação. A lesão no tornozelo foi tratada percutaneamente, tomando-se o cuidado da manutenção da divergência entre os parafusos. Nesta abordagem, foram utilizados dois parafusos corticais Orthosir (Medtronic ${ }^{\circledR}$, Dublin, Irlanda) de $3.5 \mathrm{~mm}$ em titânio no tornozelo e um parafuso cortical Orthosir em titânio na articulação tibiofibular proximal após a redução anatômica por visualização direta (-Fig. 3). Foi obtida a redução anatômica e perfeita estabilidade, preservando o arco de movimento funcional do tornozelo e do joelho, bem como o alinhamento e a rotação.

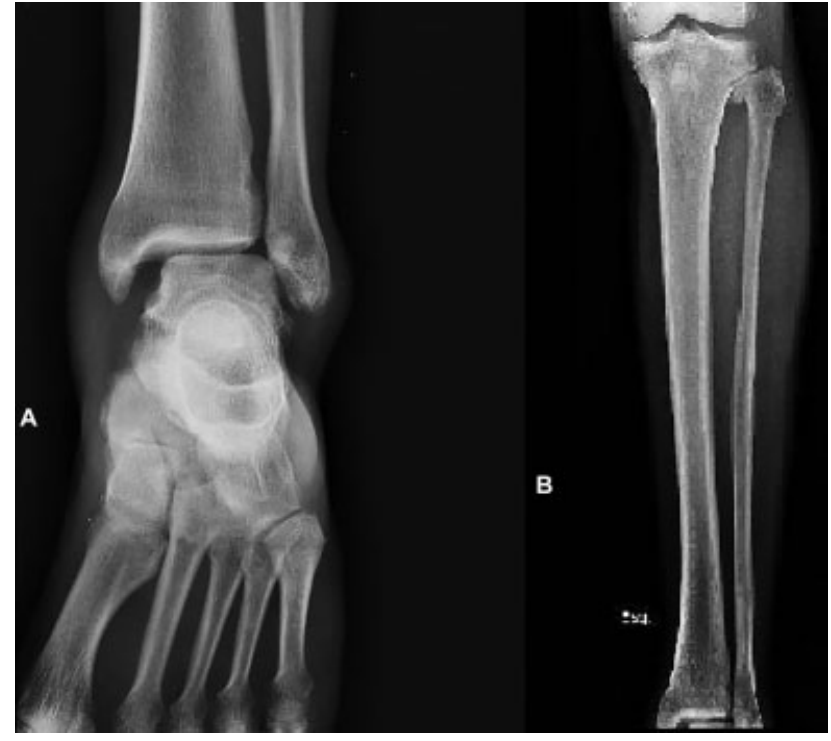

Fig. 1 Radiografias anteroposterior do tornozelo (A) e da perna (B) evidenciando disjunção da sindesmose do tornozelo e subluxação tibiofibular proximal, respectivamente.

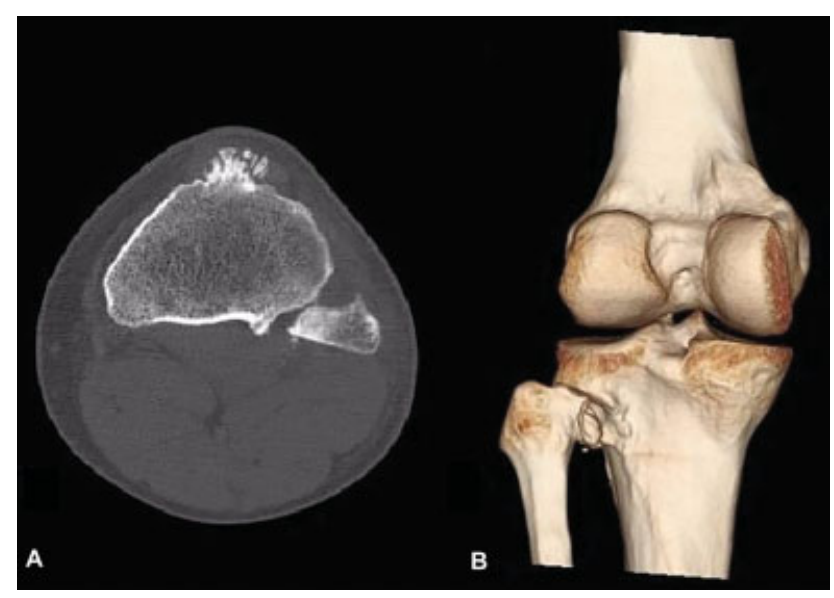

Fig. 2 Tomografia computadorizada em corte axial da articulação tibiofibular proximal (A) evidenciando subluxação tibiofibular proximal anterolateral. Visão posterior de tomografia computadorizada com reconstrução 3D de joelho $(B)$ evidenciando a subluxação anterolateral tibiofibular proximal.

A mobilização ativa e passiva do joelho e do tornozelo foi iniciada imediatamente após o ato cirúrgico. A carga também foi liberada imediatamente com uso de muleta axilar para carga parcial. Iniciaram-se exercícios de fortalecimento isométrico de coxa e perna no mesmo instante. A carga total foi liberada com 1 mês. $O$ paciente voltou às atividades laborais em 2 meses e às atividades esportivas em 3 meses. Após 6 meses de acompanhamento, o paciente possuía flexão de joelho, dorsiflexão e flexão plantar totais e sem limitações (-Fig. 4).

\section{Discussão}

A estabilidade da articulação tibiofibular proximal depende dos componentes ósseos e musculoligamentares. Apesar da fragilidade deste complexo, o deslocamento da cabeça da 


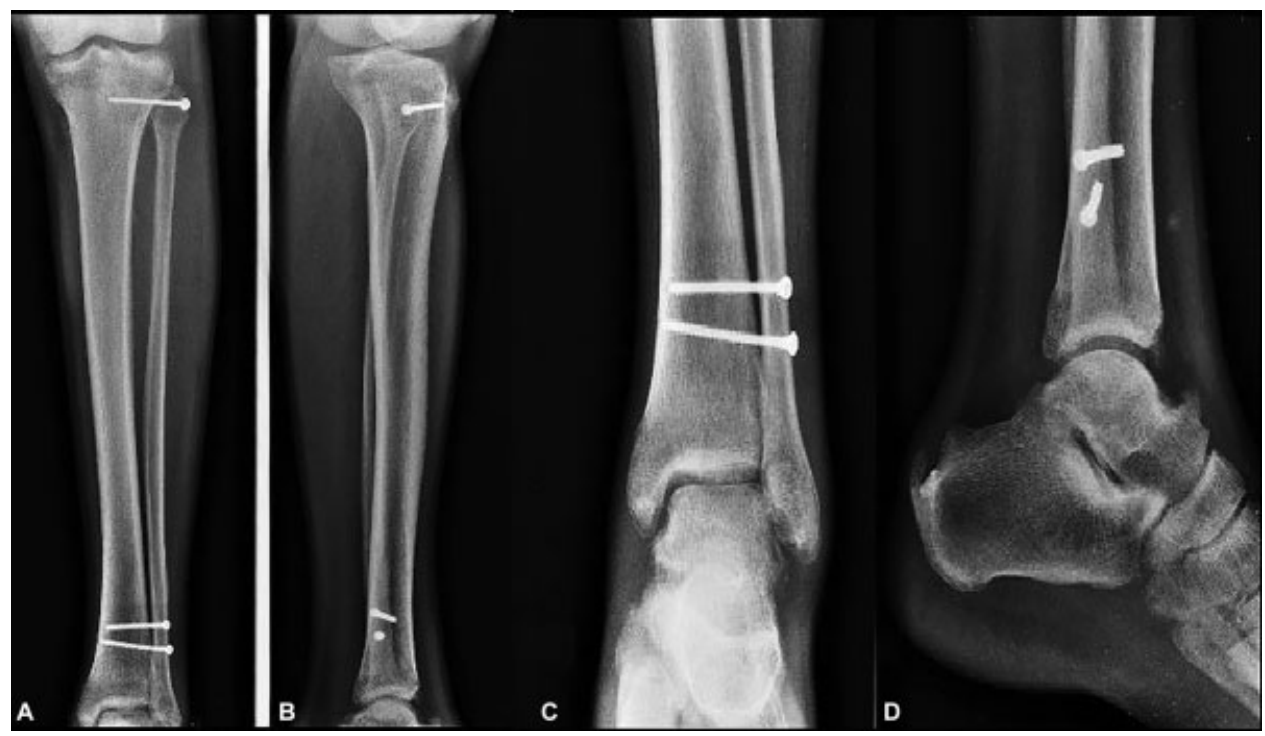

Fig. 3 Radiografia anteroposterior (A) e de perfil (B) da perna e anteroposterior (C) e de perfil (D) do tornozelo pós-cirurgia.

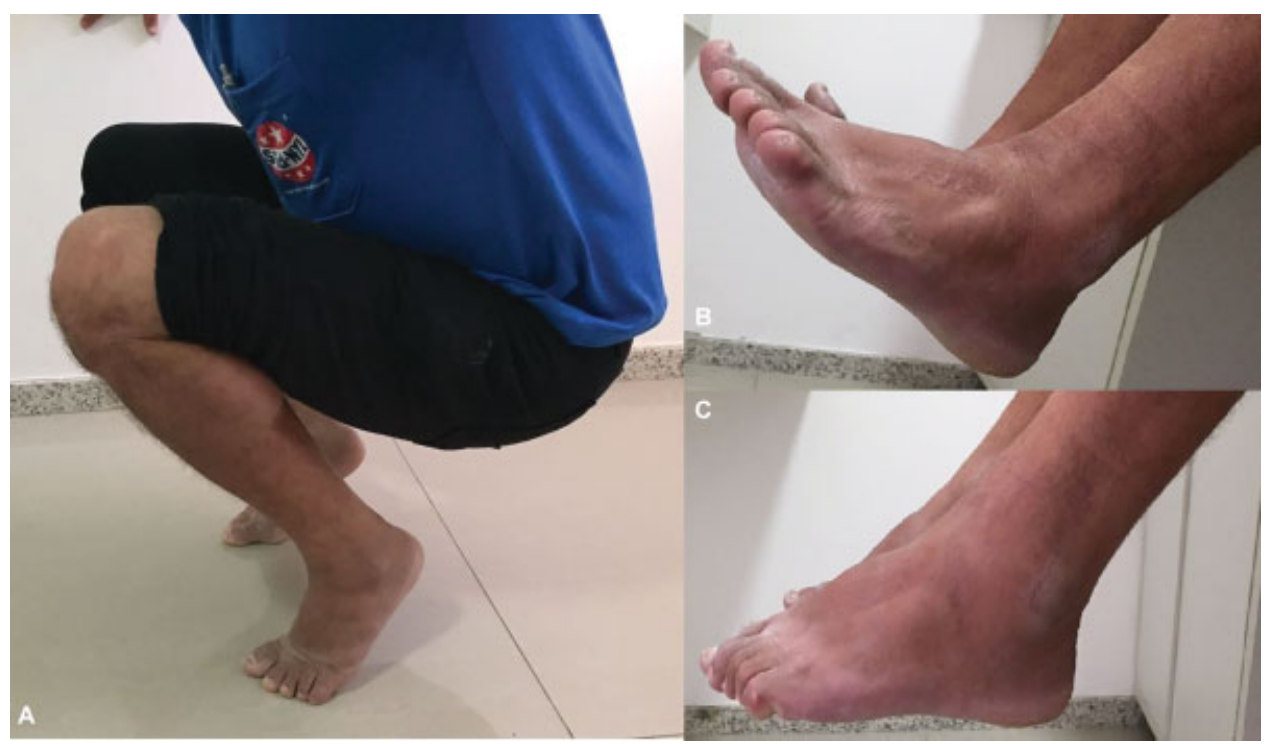

Fig. 4 Arcos de movimentos totais de joelho e tornozelo (A); Dorsiflexão total (B); Flexão plantar total (C).

fíbula é muito raro e pouco descrito na literatura. Há quatro possibilidades deste deslocamento: anterolateral (mais frequente), posteromedial, superior, e subluxação atraumática. ${ }^{5}$

Já a lesão da sindesmose tibiofibular distal é uma injúria frequente nas emergências devido a traumas torcionais no tornozelo. Entretanto, a lesão isolada do complexo ligamentar sem fratura da fíbula é rara. ${ }^{3}$

Assim, a lesão variante de Maisonneuve com luxação tibiofibular proximal é uma entidade rara que requer cautela no diagnóstico. Foi pesquisado nas bases de dados Bireme, PubMed e Lilacs, no mês de janeiro de 2018, encontrando-se apenas 4 casos descritos. ${ }^{2,4,6,7} \mathrm{~A}$ incidência variou entre traumas de alta e baixa energia, mas todos os pacientes foram abordados cirurgicamente com bons resultados funcionais e restauração completa do arco de movimento do membro.
No presente caso relatado, o paciente foi tratado com redução cirúrgica direta para luxação tibiofibular proximal e redução e fixação percutânea no tornozelo. Reportamos também a necessidade de liberar imediatamente a movimentação ativa e passiva além de carga com apoio parcial nestes pacientes para se obter bons resultados funcionais na reabilitação dos mesmos.

\section{Conflitos de interesses}

Os autores declaram não haver conflitos de interesses.

\section{Referências}

1 Ibrahim T, Beiri A, Azzabi M, Best AJ, Taylor GJ, Menon DK. Reliability and validity of the subjective component of the American Orthopaedic Foot and Ankle Society clinical rating scales. J Foot Ankle Surg 2007;46(02):65-74 
342 Lesão variante de Maisonneuve Alencar Neto et al.

2 Corrigan C, Asbury B, Alvarez RG, Nowotarski P. Dislocation of the proximal and distal tibiofibular syndesmotic complex without associated fracture: case report. Foot Ankle Int 2011;32(10): 1009-1011

3 Hinds RM, Tran WH, Lorich DG. Maisonneuve-hyperplantarflexion variant ankle fracture. Orthopedics 2014;37(11):e1040-e1044

4 Bissuel T, Gaillard F, Dagneaux L, Canovas F. Maisonneuve equivalent injury with proximal tibiofibular joint dislocation: case report and literature review. J Foot Ankle Surg 2017;56(02):404-407
5 Sreesobh KV, Cherian J. Traumatic dislocations of the proximal tibiofibular joint: a report of two cases. J Orthop Surg (Hong Kong) 2009;17(01):109-111

6 Kumar G, Sankar B, Anand S, Murali SR. Superior tibiofibular joint disruption - As a variant of Maisonneuve injury. Foot Ankle Surg 2004;10(01):41-43

7 Levy BA, Vogt KJ, Herrera DA, Cole PA. Maisonneuve fracture equivalent with proximal tibiofibular dislocation. A case report and literature review. J Bone Joint Surg Am 2006;88(05):1111-1116 